\title{
Treating chronic hepatitis C in general practice
}

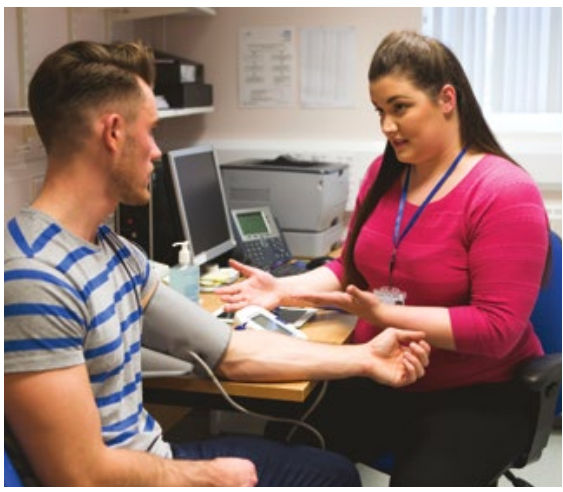

CPD

Kate Muller, Mohamed Hasan

\section{Background \\ Hepatitis $\mathrm{C}$ virus (HCV) infection continues to result in significant morbidity and mortality in Australia. Eradication of $\mathrm{HCV}$ remains a challenge, with many patients unaware of their infection. With the new era of direct-acting antivirals (DAAs), higher viral eradication rates are attainable, and access to treatment can be expanded by treating most patients with $\mathrm{HCV}$ in general practice, moving away from the traditional model of treatment by a gastroenterologist, hepatologist or infectious diseases physician. Currently available DAAs are pan-genotypic, well tolerated and safe; hence, HCV treatment can be easily undertaken in general practice.}

\section{Objective}

The aim of this article is to highlight how to use pan-genotypic DAAs and when to consider referral for a specialist opinion.

\section{Discussion}

Most patients with HCV can be treated in general practice, increasing the number of patients who have access to treatment and hence reducing the likelihood of progression to advanced liver disease in these patients, as well as advancing progress towards $\mathrm{HCV}$ eradication in Australia.
HEPATITIS C VIRUS (HCV) infection is a major cause of chronic liver disease. According to the World Health Organization, an estimated 71 million people have chronic HCV infection globally, ${ }^{1}$ many of whom are unaware of their infection. According to the latest statistics released by the Kirby Institute, chronic HCV infection remains a major public health challenge in Australia, as it affected approximately 143,580 people at the end of $2018,{ }^{2}$ a significant number of whom may be unaware of the infection. Acute infection is usually asymptomatic and progresses to chronic disease in approximately $75 \%$ of cases. These individuals are then at risk of progressive liver disease leading to cirrhosis, liver failure and hepatocellular carcinoma (HCC). With the introduction of the newly developed direct-acting antivirals (DAAs), HCV has become curable, with very well tolerated and efficacious regimens. $\mathrm{HCV}$ eradication or sustained virological response (SVR) rates of $>95 \%$ have been reported for all HCV genotypes. ${ }^{3} \mathrm{HCV}$ eradication is associated with normalisation of liver enzymes, improvement or regression of liver necroinflammation and fibrosis, and improvement in liver function. The risk of HCC, although not eliminated, is also significantly reduced.
The recommendations presented in this article are primarily based on evidence-based guidelines, published in Australia by the Gastroenterological Society of Australia (GESA), ${ }^{4}$ in addition to international guideline recommendations from the European Association for the Study of the Liver ${ }^{5}$ and the American Association for the Study of Liver Diseases. ${ }^{6}$

\section{Epidemiology of HCV in Australia}

It has been noted that the incidence of new HCV infections in Australia has been declining in recent years. This is likely due to a reduction in the prevalence of injecting drug use and improved harm reduction measures among people who inject drugs. ${ }^{4}$ A recent decline in the incidence of HCV infection may be attributed to the unrestricted availability of DAA therapy in Australia since 2016. ${ }^{7}$ Approximately 82,280 people received HCV treatment with DAAs between March 2016 and December 2019, but treatment uptake has been declining. ${ }^{8}$

$\mathrm{HCV}$ treatment in Australia is unique in that general practitioners (GPs) have been able to prescribe DAAs since their listing on the Pharmaceutical Benefits Scheme (PBS) in March 2016, and now represent an increasing proportion of prescribers. 


\section{Screening and diagnosis of HCV}

A major barrier to HCV elimination still results from the fact that a substantial proportion of patients with chronic $\mathrm{HCV}$ infection are unaware of their infection. Screening of populations at risk of $\mathrm{HCV}$ infection (Box 1) is likely to identify the majority of those individuals.

Screening for $\mathrm{HCV}$ infection is based on the detection of anti-HCV antibodies (serology testing), which indicate viral exposure. If positive, current $\mathrm{HCV}$ infection needs to be confirmed by a polymerase chain reaction (PCR) assay for HCV RNA. HCV is a notifiable disease and should be reported to the relevant Department of Health/Communicable Disease Control branch by the doctor requesting the test. The pathway for notification varies between states and territories; it is important that GPs are familiar with local requirements.

Given that a proportion of individuals with acute $\mathrm{HCV}$ will clear the virus

\section{Box 1. Population groups that should be offered screening for hepatitis $C$ virus (HCV) ${ }^{4}$}

- People who inject drugs or who have ever injected drugs

- People in custodial settings

- People with tattoos or body piercings

- People who received a blood transfusion or organ transplant before 1990

- People with coagulation disorders who received blood products or plasma-derived clotting factor treatment products before 1993

- Children born to mothers who are HCV positive

- People infected with human immunodeficiency virus (HIV) or hepatitis $B$ virus

- Sexual partners of a person who is HCV positive (individuals at higher risk of sexual transmission include men who have sex with men and people with HCV-HIV coinfection)

- People with evidence of liver disease (persistently elevated alanine aminotransferase level)

- People who have had a needle-stick injury

- Migrants from high-prevalence regions (Egypt, Pakistan, Mediterranean and Eastern Europe, Africa and Asia) spontaneously within six months, PBS eligibility requires evidence of chronic infection, which can be documented by a repeated positive serology test, along with a positive HCV RNA.

\section{Work-up of individuals who are HCV positive}

All individuals undergoing treatment for HCV should undergo a work-up prior to treatment initiation. This includes:

- thorough medical history to estimate the possible infection duration and identify underlying risk factors for $\mathrm{HCV}$, prior treatments for HCV and other risk factors for liver disease (eg alcohol intake and obesity)

- social history to assess likelihood of adherence and ongoing risk factors for reinfection

- detailed drug history (including herbal and over-the-counter medications), to assess drug-drug interactions

- physical examination to assess for any stigmata of chronic liver disease

- basic blood investigations including full blood examination, electrolyte panel, liver function tests (LFTs) and international normalised ratio (INR)

- pregnancy tests performed in women of childbearing age

- HCV genotyping - this is no longer a prerequisite for treatment initiation; however, it is recommended that $\mathrm{HCV}$ genotype be determined as this can help in differentiating relapse from reinfection among people with a high risk of reinfection

- exclusion of other infections, notably hepatitis B virus (HBV), human immunodeficiency virus (HIV) and hepatitis A virus

- assessment of liver fibrosis non-invasively with the following methods

- serum markers, such as aspartate aminotransferase to platelet ratio index (APRI) or Hepascore. APRI calculators are available online, with a score of $<0.5$ indicating significant fibrosis is unlikely, while $>1.0$ indicates significant fibrosis or cirrhosis is likely to be present. Hepascore is a score based on age, sex and biochemical serum markers; it can be requested via pathology services.

- elastography, such as transient elastography (FibroScan) or shear wave elastography.

\section{Goal of HCV treatment}

The goal of treatment is cure, or SVR, defined as undetectable plasma HCV RNA measured at least 12 weeks after treatment cessation.

\section{Indications for treatment}

Treatment should be offered to all individuals with confirmed $\mathrm{HCV}$, except those with a limited life expectancy. ${ }^{4}$

\section{Direct-acting antivirals}

DAAs are highly effective and well tolerated and have a short treatment duration, particularly when compared with the previous interferonbased regimens. The availability of pan-genotypic regimens simplifies treatment choice, and there is no need to wait for HCV genotype results; most patients can be treated in primary care.

A useful tool available for GPs who do not have experience treating patients with DAAs is the remote consultation form that can be accessed via the GESA website (www.gesa.org.au/education/ clinical-information), allowing GPs to seek support from gastroenterology, hepatology or infectious diseases services while familiarising themselves with DAA treatment.

Three pan-genotypic DAA regimens are listed on the PBS, two of which are suitable for treatment of patients by GPs. These are summarised in Table 1. PBS prescription requires telephone authority, and the cirrhosis status of the patient is required for approval.

Sofosbuvir, velpatasvir plus voxilaprevir is the third pan-genotypic DAA combination available on the PBS. It was developed as a rescue regimen for individuals who fail one of the two first-line DAA regimens; hence, it is suitable for patients treated in specialised 
gastroenterology, hepatology or infectious diseases centres.

\section{Drug-drug interactions}

Concomitant medications should be determined and potential interactions assessed before starting treatment. The University of Liverpool's interaction checker is an excellent resource (www.hep-druginteractions.org).

Some common drug interactions include:

- proton pump inhibitors

- statins

- St John's wort

- ethinyloestradiol-containing contraceptives

- anti-epileptics

- amiodarone.

\section{Treatment of special populations}

Box 2 summarises the subpopulations that are not suitable for treatment in general practice and should be referred for management by the relevant specialist (eg paediatrics, hepatology, infectious diseases and liver transplantation). ${ }^{6}$

\section{Pregnancy and breastfeeding}

There are limited safety data for the use of DAAs during pregnancy ${ }^{9-11}$ or lactation. Therefore, treatment of women who are pregnant or breastfeeding is not currently recommended. Ribavirin is teratogenic.

\section{Children}

PBS criteria now allow for treatment of those aged 12 years and above with DAAs.

This sub-group of individuals who are $\mathrm{HCV}$ positive should be referred to a paediatrician with experience in HCV treatment.

\section{Non-responders}

Failure of DAA treatment is defined by detectable serum HCV RNA after treatment. This may be due to breakthrough or relapse related to medication resistance, or due to non-adherence or reinfection.

Individuals who fail first-line DAA therapy should be referred to a specialised gastroenterology, hepatology or infectious diseases centre for retreatment. Where reinfection is confirmed, treatment can be undertaken as for a treatment-naive patient.

\section{Coinfection}

Coinfection with HBV or HIV and HCV is associated with an increased rate of progression to liver cirrhosis, increased risk of hepatocellular carcinoma (HCC) and increased mortality. Patients with HCV should be tested for HBV and HIV. Patients with coinfection should be referred for treatment in specialised hepatology or infectious diseases centres.

\section{Decompensated cirrhosis}

Individuals with decompensated

liver disease should be referred for management in a specialised hepatology centre.

The Child-Pugh score (Table 2) can be used to distinguish between populations with compensated and decompensated cirrhosis.

\section{On-treatment monitoring}

In general, monitoring during treatment is not required. Pre-treatment work-up and 12-week post-treatment blood tests for LFTs and HCV PCR are recommended for patients with uncomplicated HCV. More regular monitoring may be required in some populations (eg patients with decompensated cirrhosis, those receiving ribavirin). Patients at risk of non-adherence may benefit from regular follow-up during treatment.

\section{Determining SVR}

SVR is defined as a negative HCV RNA viral load performed 12 weeks after completion of treatment. HCV antibody remains positive in cured individuals and should not be used to assess treatment response or to assess for reinfection. There is no protection against reinfection; patients at high risk of reinfection can be serially monitored with HCV PCR.

A detected HCV RNA 12 weeks post-treatment signifies failure of treatment. Non-responders should be

Table 1. Treatment regimens for hepatitis $\mathrm{C}$ virus in people who are treatment naive

\begin{tabular}{|c|c|c|c|c|}
\hline \multirow[t]{2}{*}{ Drug regimen } & \multirow[t]{2}{*}{ Dosing } & \multicolumn{2}{|c|}{ Duration (treatment naive) } & \multirow[t]{2}{*}{ Comments } \\
\hline & & No cirrhosis & Cirrhosis & \\
\hline \multirow[t]{3}{*}{$\begin{array}{l}\text { Sofosbuvir plus } \\
\text { velpatasvir }\end{array}$} & \multirow[t]{3}{*}{$\begin{array}{l}\text { One tablet, } \\
\text { once daily }\end{array}$} & \multirow[t]{3}{*}{12 weeks } & \multirow[t]{3}{*}{12 weeks } & $\begin{array}{l}\text { Consider adding weight-based ribavirin for patients } \\
\text { with genotype } 3 \text { hepatitis } \mathrm{C} \text { virus (HCV) who have } \\
\text { compensated cirrhosis. }\end{array}$ \\
\hline & & & & $\begin{array}{l}\text { Side effects include headache, fatigue, nausea and } \\
\text { nasopharyngitis. }\end{array}$ \\
\hline & & & & Of note, renal dose adjustment is no longer required. \\
\hline \multirow{3}{*}{$\begin{array}{l}\text { Glecaprevir plus } \\
\text { pibrentasvir }\end{array}$} & \multirow{3}{*}{$\begin{array}{l}\text { Three tablets, once } \\
\text { daily with food }\end{array}$} & \multirow[t]{3}{*}{ Eight weeks } & \multirow[t]{3}{*}{ Eight weeks } & Side effects include headache, fatigue and nausea. \\
\hline & & & & No renal dose adjustment is required. \\
\hline & & & & $\begin{array}{l}\text { Not recommended for individuals with } \\
\text { decompensated cirrhosis (Child-Pugh B and C). }\end{array}$ \\
\hline
\end{tabular}




\section{Table 2. Child-Pugh score}

\begin{tabular}{llll}
\hline Factor & One point & Two points & Three points \\
\hline Bilirubin (mmol/L) & $<34$ & $34-50$ & $>50$ \\
\hline Albumin (g/L) & $>35$ & $28-35$ & $<28$ \\
\hline $\begin{array}{l}\text { International } \\
\text { normalised ratio }\end{array}$ & $<1.7$ & $1.71-2.3$ & $>2.3$ \\
\hline Ascites & None & Mild & Moderate to severe \\
\hline Encephalopathy & None & Grades I-II & Grades III-IV \\
\hline & Class A & Class B & Class C \\
\hline Total points & 5-6 & $7-9$ & $10-15$ \\
\hline & Compensated & Decompensated & Decompensated \\
\hline
\end{tabular}

referred for specialist hepatology or infectious diseases management for retreatment.

\section{Post-treatment monitoring}

Screening for HCC with ultrasonography is recommended at baseline for all patients with cirrhosis. These patients require serial ultrasonography (every six months) even after achieving SVR as the risk of HCC, although reduced, is not eliminated. Patients with cirrhosis should be referred to a specialised hepatology centre for ongoing cirrhosis management and monitoring, even if SVR is achieved.

Box 2. Patients who are not suitable for hepatitis $\mathbf{C}$ virus treatment in general practice $^{6}$

- Children

- Adult patients who have any of the following characteristics:

- current or prior episode of decompensated cirrhosis, defined as Child-Pugh score $\geq 7$

- prior hepatitis $C$ virus infection and treatment (excluding failed peginterferon/ribavirin regimens)

- end-stage renal disease

- human immunodeficiency virus or hepatitis B surface antigen

- current pregnancy

- hepatocellular carcinoma

- liver transplantation
Endoscopies might be required to rule out oesophageal varices, along with bone mineral density testing to assess for osteoporosis.

People who do not have cirrhosis and who have normal LFT results after SVR (alanine aminotransferase $\leq 30 \mathrm{U} / \mathrm{L}$ in males, $\leq 19 \mathrm{U} / \mathrm{L}$ in females) do not require further follow-up. If LFTs do not normalise with successful treatment of $\mathrm{HCV}$, the patient should be evaluated for other causes of liver disease and referred for review by a hepatologist.

\section{Conclusion}

HCV treatment should be offered to all individuals with chronic HCV, established by positive HCV RNA. The Australian model is unique in providing GP access to DAAs, in an attempt to increase feasibility, reduce waiting times and thereby increase the number of individuals receiving treatment. The safety of the pan-genotypic regimens and ease of dosing have made HCV treatment much more simplified, compared with the previous era of interferon and early genotype-specific DAA regimens. These factors have made it safe and feasible for GPs to treat both patients who do not have cirrhosis and those with compensated cirrhosis. The latter require ongoing follow-up even after achieving SVR and, along with patients with decompensated cirrhosis and other subpopulations, should be referred for specialist hepatology care.

\section{Key points}

- Many people with HCV are undiagnosed, and at-risk populations should be screened for infection.

- DAA therapy for HCV is safe, well tolerated and efficacious.

- Treatment of most patients, including those with compensated cirrhosis, can be undertaken in general practice.

- Specific populations of patients with HCV should be referred for care to the relevant specialist.

\section{Resources}

- Gastroenterological Society of Australia, www.gesa.org.au

- Australasian Society for HIV, Viral Hepatitis and Sexual Health Medicine, www.ashm.org.au

- Hepatitis Australia, www.hepatitisaustralia.com

- Australasian Hepatology Association, www.hepatologyassociation.com.au

\section{Authors}

Kate Muller MBBS, PhD, FRACP, Staff Specialist, Flinders Medical Centre, Bedford Park, SA; Senior Lecturer, Flinders University, Bedford Park, SA Mohamed Hasan BSc, MD, Hepatology Fellow, Flinders Medical Centre, Bedford Park, SA

Competing interests: $\mathrm{KM}$ has received honoraria from Gilead and Novartis for advisory board participation and speaking. She is a member of the Gastroenterological Society of Australia Liver Faculty. Funding: None.

Provenance and peer review: Commissioned externally peer reviewed.

Correspondence to:

kate.muller@sa.gov.au

\section{References}

1. World Health Organization. Global hepatitis report 2017. Geneva, CH: WHO, 2017.

2. Kirby Institute. National update on HIV, viral hepatitis and sexually transmissible infections in Australia: 2009-2018. Sydney, NSW: University of NSW, 2020.

3. Zeuzem S, Foster GR, Wang S, et al. Glecaprevirpibrentasvir for 8 or 12 weeks in HCV genotype 1 or 3 infection. N Engl J Med 2018;378(4):354-69. doi: 10.1056/NEJMoa1702417.

4. Hepatitis C Virus Infection Consensus Statement Working Group. Australian recommendations for the management of hepatitis $\mathrm{C}$ virus infection: A consensus statement (June 2020). Melbourne, Vic: Gastroenterological Society of Australia, 2020.

5. European Association for the Study of the Liver. EASL recommendations on treatment of hepatitis C: Final update of the series. J Hepatol 2020;73(5):1170-218. doi: 10.1016/j. jhep.2020.08.018.

6. Ghany MG, Morgan TR; AASLD-IDSA Hepatitis C Guidance Panel. Hepatitis C guidance 2019 
update: American Association for the Study of Liver Diseases - Infectious Diseases Society of America recommendations for testing, managing, and treating hepatitis $\mathrm{C}$ virus infection. Hepatology 2020;71(2):686-721. doi: 10.1002/hep.31060.

7. Kirby Institute. HIV, viral hepatitis and sexually transmissible infections in Australia: Annual surveillance report 2018. Sydney, NSW: University of NSW, 2018.

8. Burnet Institute and Kirby Institute. Australia's progress towards hepatitis $\mathrm{C}$ elimination: Annual report 2020. Melbourne, Vic: Burnet Institute, 2020.

9. Yattoo GN. Treatment of chronic hepatitis $\mathrm{C}$ with ledipasvir/sofosbuvir combination during pregnancy [Abstract]. Hepatol Int 2018;12 Supp 2:S292-93.

10. Chappell CA, Scarsi KK, Kirby BJ, et al. Ledipasvir plus sofosbuvir in pregnant women with hepatitis $C$ virus infection: A phase 1 pharmacokinetic study. Lancet Microbe 2020;1(5):e200-08. doi: 10.1016/ S2666-5247(20)30062-8.

11. AbdAllah M, Alboraie M, Abdel-Razek W, et al. Pregnancy outcome of anti-HCV direct-acting antivirals: Real-life data from an Egyptian cohort. Liver Int 2021;41(7):1494-97. doi: 10.1111/liv.14913. 